\title{
TRANSFORMAÇÃO DE COORDENADAS COM MODELAGEM DE DISTORÇÕES ENTRE SAD69 E SIRGAS2000 COM O USO DE THIN-PLATE SPLINES
}

Coordinate transformation with distortion modeling between SAD69 and SIRGAS2000 by using Thin-Plate Splines

\author{
JOÃO PAULO MAGNA JÚNIOR ${ }^{1}$ \\ PAULO DE OLIVEIRA CAMARGO ${ }^{2}$ \\ MAURÍCIO GALO ${ }^{2}$
}

\author{
${ }^{1}$ Instituto Federal de Educação, Ciências e Tecnologia de Goiás \\ Campus de Goiânia - Goiás - Brasil \\ ${ }^{2}$ UNESP - Univ. Estadual Paulista - Campus de Presidente Prudente \\ Programa de Pós-Graduação em Ciências Cartográficas - PPGCC \\ Presidente Prudente - São Paulo - Brasil \\ joao.paulo@ifg.edu.br, \{paulo, galo\}@fct.unesp.br
}

\begin{abstract}
RESUMO
Diversos países têm passado por processos de mudança na estrutura geodésica fundamental, convergindo na adoção de referenciais geodésicos geocêntricos. No Brasil, a adoção do SIRGAS2000 envolve a existência conjunta de duas realizações do sistema Córrego Alegre, duas realizações do sistema SAD69 e uma realização do sistema SIRGAS2000. Para o aproveitamento de produtos nos referenciais antigos são necessários métodos para transformação de coordenadas entre os referenciais existentes. Neste contexto, este artigo apresenta uma solução para a transformação de coordenadas entre referenciais, baseado em Thin-Plate Splines (TPS), que reúne características para a modelagem de dados provenientes de uma transformação linear, juntamente com distorções de natureza não linear. $O$ modelo foi desenvolvido para trabalhar com coordenadas cartesianas tridimensionais, sendo apresentados neste artigo resultados com dados simulados bem como com dados do SGB (Sistema Geodésico Brasileiro). Em pontos de verificação em SAD69 (realização de 1996 - SAD69/96) foram obtidos valores de RMSE (Root Mean Square Error) de 78,2 $\mathrm{mm}$ em latitude e $67,5 \mathrm{~mm}$ em longitude após a
\end{abstract}


transformação para SIRGAS2000. Na comparação entre o modelo TPS e o proporcionado pelo ProGriD os indicadores estatísticos foram reduzidos em $97 \%$ ao utilizar o modelo TPS, indicando que o uso deste modelo é promissor, possibilitando uma maior qualidade no processo de transformação de coordenadas e modelagem da distorção entre referenciais geodésicos.

Palavras-chave: Thin-Plate Splines; Modelagem de Distorções; Transformação de Coordenadas; Referenciais Geodésicos.

\section{ABSTRACT}

Several countries have been passed by change processes in their fundamental geodesic structure with the focus on the adoption of geocentric reference systems. In Brazil, the adoption of the SIRGAS2000 evolves the coexistence of two realizations from the Córrego Alegre system, two realizations from the SAD69 system and one realization from the SIRGAS2000 system. To make use of products in the old reference systems, methods of coordinate transformation between the existent reference frames are necessary. So, in this paper one solution for the transformation between coordinates from different reference frames, based on Thin-Plate Splines (TPS), that allows the estimation of parameters from one linear transformation and also one non-linear model is presented. The TPS model was developed to work with tridimensional coordinates and in this paper the results and analysis are performed with simulated data and also with data from the official Brazilian Geodetic System (SGB). In the check points from SAD69 stations (realization of 1996 - SAD69/96), the values of RMSE obtained were of $78,2 \mathrm{~mm}$ in latitude and $67,5 \mathrm{~mm}$ in longitude, before the transformation to the SIRGAS2000. In the comparison between the TPS model and ProGriD (Brazilian software provided by IBGE), the statistical indicators were reduced in $97 \%$, by using the TPS model. Based in the obtained results from real dataset, the TPS model appears to be promising, since it allows improving the quality of transformation process with simultaneous distortion modeling.

Keywords: Thin-Plate Splines; Distortion Modeling; Coordinate Transformation; Reference Systems.

\section{INTRODUÇÃO}

$\mathrm{Na}$ busca por acompanhar a tendência mundial e a evolução tecnológica, o Sistema Geodésico Brasileiro (SGB) vem se adaptando ao longo dos anos, procurando prover para a comunidade usuária dados para posicionamento geodésico e mapeamento de maior acurácia. A mais atual e significante mudança ocorreu em 2005, com a adoção do SIRGAS2000 (Sistema de Referência Geocêntrico para as Américas) oficialmente como novo referencial geodésico.

A disponibilização, tanto de novas materializações para referenciais já existentes quanto de novos referenciais, culmina com a necessidade de métodos para relacionar as diferentes coordenadas entre estas realizações. A transformação de 
coordenadas e a modelagem de distorções entre referenciais passaram a ser fonte de pesquisa e uma preocupação latente quando se trata de dados georreferenciados. Estudos realizados pelo Grupo de Trabalho 3 (GT3) do Projeto Mudança do Referencial Geodésico (PMRG) e do Projeto da Infraestrutura Geoespacial Nacional (PIGN) colaboraram com a disponibilização de um aplicativo para a transformação de coordenadas entre os referenciais utilizados no Brasil (OLIVEIRA et al., 2008 e MAGNA JÚNIOR, 2009). Em dezembro de 2008, o IBGE passou a disponibilizar o aplicativo denominado ProGriD em um esforço de prover para a comunidade de usuários de dados geoespaciais de um sistema computacional que facilite a transição para o SIRGAS2000 (IBGE, 2009).

As características próprias da rede planimétrica Brasileira, sua grande extensão e os processos de levantamento e ajuste de suas coordenadas ao longo do tempo, a torna diferenciada de outras redes no mundo. Os efeitos do comportamento não homogêneo das distorções, assim como a variação da sua magnitude ao longo da rede, podem impactar negativamente o processo de modelagem e merecem investigações. O processo de mudança de referenciais ou da disponibilização de novas realizações para os referenciais já existentes é constante. Volumes cada vez maiores de dados, com melhor qualidade se tornam disponíveis a cada momento, logo, deve-se ter uma rede geodésica com qualidade compatível. Nesse sentido, a pesquisa de métodos que proporcionem um relacionamento de qualidade adequada entre diferentes realizações disponíveis torna-se necessária e de relevância tanto operacional quanto científica.

A metodologia proposta nesse artigo se fundamenta na modelagem baseada em funções de mapeamento por Thin-Plate Spline (TPS), usadas para determinar distorções relativas em dois conjuntos de pontos de controle correspondentes. As características das funções TPS são ideais para a transformação de coordenadas e modelagem de distorções, pois é possível a modelagem a partir de dados homólogos resultantes de uma transformação geométrica (translação, rotação, não ortogonalidade e fator de escala) e sujeitos a distorções de natureza não linear. Baseado neste princípio foi expandido o modelo TPS tradicional para a utilização de coordenadas geodésicas cartesianas tridimensionais na estimativa dos coeficientes TPS seguida da avaliação da qualidade desta modelagem.

\section{TRANSFORMAÇÃO DE COORDENADAS E MODELAGEM DE DISTORÇÕES NA REDE GEODÉSICA BRASILEIRA}

No Brasil, diferentes metodologias foram utilizadas no processo de transformação de coordenadas entre referenciais geodésicos. Em IBGE (1983) são sugeridas as equações diferenciais simplificadas de Molodenski para a transformação de coordenadas geodésicas curvilíneas entre os sistemas Córrego Alegre e SAD69. Com a adoção do SIRGAS2000, foram publicados em IBGE (2005) parâmetros para a transformação de coordenadas geodésicas cartesianas entre SAD69 e SIRGAS2000, utilizando a abordagem cartesiana clássica. Nesse processo, eram estimados parâmetros de transformação (especificamente 
translações) entre os sistemas de referência, considerando o paralelismo dos eixos de suas superfícies de referência.

Em 2005, o IBGE disponibilizou o aplicativo denominado TCGEO, o qual possibilitava aos usuários a conversão de coordenadas entre os referenciais SAD69 e SIRGAS2000. A conversão também se baseava somente na aplicação de parâmetros de translação e o aplicativo apresentava como saída um resíduo da transformação, que servia como um indicativo do valor de distorção no ponto considerado. Para o cálculo dos resíduos, dois fatores eram considerados: a posição geográfica do ponto e a realização da qual o ponto pertence. A distinção quanto à origem das coordenadas era feita em termos de rede GPS, rede clássica SAD69 e rede clássica SAD69/96.

A mudança mais recente no que se refere à conversão de referenciais no SGB ocorreu em dezembro de 2008, com o lançamento do ProGriD (IBGE, 2009). O principal diferencial do ProGriD consiste na incorporação da modelagem das distorções no processo de conversão de coordenadas. Para realizar as transformações, o ProGriD utiliza grades no formato NTv2 (National Transformation version 2), um pacote de aplicativos e procedimentos desenvolvido no Canadá e utilizado em vários países para a conversão de referenciais. O modelo de distorção utilizado pelo NTv2 é composto por: um modelo global, que utiliza um polinômio complexo para modelar simultaneamente as distorções em latitude e longitude; e um modelo local, que trata individualmente a modelagem nas componentes latitude e longitude em função das distorções residuais globais (JUNKINS e ERICKSON, 1996). As distorções modeladas são disponibilizadas em um formato padrão do NTv2, compatível com diversos programas computacionais de geoprocessamento. As grades de transformação NTv2 utilizadas no ProgriD são geradas a partir de estações homólogas nas seguintes materializações (IBGE, 2009): Córrego Alegre (1961), Córrego Alegre (1970+1972), SAD69 Rede Clássica e SAD69/96 Rede Clássica. Os pontos determinados com técnicas de posicionamento por satélites não são utilizados no conjunto de estações de cálculo do SAD69, uma vez que é dado um tratamento diferenciado para estas estações, as quais foram denominadas no ProGriD de SAD69 Técnica Doppler ou GPS. O tratamento diferenciado das estações em função da técnica de levantamento se deve ao padrão distinto das distorções que, nas estações determinadas por posicionamento GNSS apresentam comportamento mais homogêneo e de menor magnitude quando comparadas com as estações determinadas por métodos clássicos de levantamento.

Além dos procedimentos recomendados pelo IBGE, diferentes soluções e estudos foram publicados com o propósito de contribuir para o processo de conversão de referenciais no Brasil, dentre os quais se destacam: Costa (1999), Magna Júnior (2007, 2012), Oliveira (1998) e Pino e Firkowski (2009). 


\section{THIN-PLATE SPLINES: DEFINIÇÃO E CARACTERIZAÇÃO MATEMÁTICA}

Thin-Plate Splines apresentam características que as tornam aplicáveis em grande parte dos problemas de modelagem, pois possibilitam a decomposição da modelagem em uma transformação linear e uma componente de distorções não lineares. O conceito de TPS se baseia na minimização da energia de curvatura de uma placa delgada de metal fixa a alguns pontos amostrais. Sua formulação garante restrições de que a superfície interpolante apresente mínima energia de deformação e que seja suave. De acordo com Bookstein (1989), para uma placa delgada de metal, sujeita a uma curvatura suave, a energia de curvatura em um ponto com coordenadas $(x, y)$ é dada por:

$$
I_{f}=\iint_{R 2}\left(\left(\frac{\partial^{2} z}{\partial x^{2}}\right)^{2}+2\left(\frac{\partial^{2} z}{\partial x \partial y}\right)^{2}+\left(\frac{\partial^{2} z}{\partial y^{2}}\right)^{2}\right) d x d y
$$

A função spline $f(x, y)$ que minimiza a energia de curvatura $I_{f}$ é dada por:

$$
f(x, y)=a+b x+c y+\sum w_{i} U(r),
$$

onde:

$a, b, c$ e $w_{i}(i=1, \ldots, n)$ - coeficientes da Thin-Plate Spline;

$U(\mathrm{r})$ - função de base radial; e

$r$ - distância do ponto $(x, y)$ ao i-ésimo ponto de controle.

A função TPS é, como se pode notar pela Equação 2, formada a partir da combinação linear de funções radiais $(U(\mathrm{r}))$, onde os valores da função são obtidos pelas diferenças (ou distâncias) entre as coordenadas dos pontos de controle (utilizados na geração do modelo). A função de base radial $U(r)$ apresenta um comportamento linear à medida que os pontos analisados se afastam da origem, a qual é dada pelos pontos de controle. Embora sejam funções de interpolação globais, as influências das deformações locais tendem a diminuir com o afastamento em relação aos pontos de controle, conforme destaca Castanho (1997).

Uma das aplicações da TPS é na geração de mapas $R^{2}$ para $R^{2}$ relacionando dois conjuntos de pontos homólogos. A função de mapeamento TPS pode ser decomposta em uma transformação linear (transformação afim) e uma parcela de distorções não lineares. Em grande parte das soluções são geradas superfícies de interpolação $f(x, y)$ em função das coordenadas bidimensionais planas $(x, y)$. A formulação e detalhes sobre as funções de mapeamento 2D podem ser encontradas em Bookstein (1989).

A base das análises e da formulação da TPS está no estudo de uma função de base radial $U$, a qual é dependente do espaço dos números reais a ser utilizado. De 
acordo com Bookstein (1989) e Tsai et al. (2000), na modelagem com coordenadas no espaço bidimensional $\left(R^{2}\right)$ a função $U$ é dada pela Equação 3; já no caso tridimensional $\left(R^{3}\right), U$ é dada pela Equação 4, respectivamente:

$$
\begin{gathered}
z(x, y)=U(r)=-r^{2} \log \left(r^{2}\right), \\
z(x, y, z)=U(r)=\|r\|_{2},
\end{gathered}
$$

onde $r$ é a distância euclidiana à origem do sistema cartesiano, definida pelas coordenadas de cada ponto de controle e é dada pela norma-2 de $r$ na Equação 4.

A combinação linear de múltiplos termos de $U(r)$ é utilizada para modelar uma placa delgada de metal que se estende em todas as direções, conforme a definição de TPS. A partir da função de base radial e da Equação 2 é possível definir as funções de mapeamento TPS em $X, Y$ e $Z$, dadas pelas equações:

$$
\begin{aligned}
& X_{2}=a_{0}+a_{1} X_{1}+a_{2} Y_{1}+a_{3} Z_{1}+\sum_{i=1}^{n} u_{i} U\left(r_{i}\right), \\
& Y_{2}=a_{4}+a_{5} X_{1}+a_{6} Y_{1}+a_{7} Z_{1}+\sum_{i=1}^{n} v_{i} U\left(r_{i}\right), \\
& Z_{2}=a_{8}+a_{9} X_{1}+a_{10} Y_{1}+a_{11} Z_{1}+\sum_{i=1}^{n} w_{i} U\left(r_{i}\right),
\end{aligned}
$$

sendo:

$X_{2}, Y_{2}, Z_{2}$ - coordenadas cartesianas geodésicas na realização de destino;

$X_{1}, Y_{1}, Z_{1}$ - coordenadas cartesianas geodésicas na realização de origem; $a_{0}, a_{1}, \ldots, a_{11}, u_{1}, \ldots, u_{n}, v_{1}, \ldots, v_{n}, w_{1}, \ldots, w_{n}$ - coeficientes das funções TPS em $X, Y$ e $Z$

$U\left(r_{i}\right)$ - Equação 4, sendo $r_{i}$ a distância Euclidiana no espaço tridimensional entre um determinado ponto e cada um dos $n$ pontos de controle.

Para a determinação dos coeficientes do modelo TPS é necessário a resolução do sistema de equações formado pelas Equações 5 e pelas injunções dadas pelas equações:

$$
\begin{aligned}
\sum_{i=1}^{n} u_{i}=0 & \sum_{i=1}^{n} v_{i}=0 & \sum_{i=1}^{n} w_{i}=0 \\
\sum_{i=1}^{n} u_{i} X_{i}=0 & \sum_{i=1}^{n} u_{i} Y_{i}=0 & \sum_{i=1}^{n} u_{i} Z_{i}=0 \\
\sum_{i=1}^{n} v_{i} X_{i}=0 & \sum_{i=1}^{n} v_{i} Y_{i}=0 & \sum_{i=1}^{n} v_{i} Z_{i}=0 \\
\sum_{i=1}^{n} w_{i} X_{i}=0 & \sum_{i=1}^{n} w_{i} Y_{i}=0 & \sum_{i=1}^{n} w_{i} Z_{i}=0
\end{aligned}
$$


O conjunto de Equações 6 assegura o crescimento linear da TPS à medida que o ponto de avaliação da função se torna mais distante de um ponto de controle (CASTANHO, 1997) e garante que o sistema linear a ser solucionado tenha número de equações iguais ao número de incógnitas, independentemente do número de pontos de controle. O sistema solucionado individualmente para cada coordenada é formado por $n+12$ equações e $n+12$ incógnitas, sendo $n$ o número de pontos de controle.

No caso específico do SGB, as estações estão distribuídas irregularmente ao longo do território brasileiro, tanto em termos de espaçamento quanto de densidade das estações. Um problema que pode ocorrer na modelagem por TPS é que dois pontos muito próximos podem ter distâncias relativamente pequenas com relação aos demais pontos da rede. Isso implica na existência de linhas e/ou colunas da matriz $A$ (matriz dos coeficientes) com dependência linear, provocando problemas de condicionamento, como pode-se ver em Magna Júnior (2012).

Como forma de melhorar o condicionamento da matriz $A$ foi adotado um limiar de distância para pontos próximos. Um pré-processamento é realizado nos pontos de controle para identificar pontos que estejam próximos a uma distância igual ou inferior ao limiar estabelecido. Uma vez identificados os pontos que atendem a essa condição, apenas um dos pontos é mantido no conjunto de dados e o outro é descartado. Em Magna Júnior (2012), experimentos foram realizados para estabelecer o limiar de distância, levando em consideração o número de pontos excluídos, a característica e o condicionamento da matriz $A$ para cada um dos limiares analisados. Considerando as estações do SGB, o limiar que melhor atendeu aos critérios analisados foi o de $1 \mathrm{~km}$. Pode-se pensar que ao eliminar pontos de uma rede a qualidade da modelagem pode ser prejudicada, o que é um aspecto a ser considerado. No entanto, experimentos realizados mostraram que, assumindo que as redes geodésicas apresentam localmente uma deformação homogênea, esta eliminação não prejudica os resultados da modelagem, uma vez que a modelagem TPS gera uma superfície suave e fixa nos pontos de controle utilizados.

\section{MÉTODO PROPOSTO PARA TRANSFORMAÇÃO DE COORDENADAS E MODELAGEM DE DISTORÇÕES}

A metodologia proposta se baseia na utilização de coordenadas geodésicas cartesianas $(X, Y$ e $Z)$ para a modelagem por TPS. Embora as coordenadas de estações do SGB sejam disponibilizadas em latitude, longitude e altitude geométrica, para evitar problemas de distorção, por exemplo, decorrente da representação bidimensional dos dados em uma projeção, optou-se por trabalhar com coordenadas geodésicas cartesianas obtidas a partir das coordenadas geodésicas.

A fase inicial do desenvolvimento da metodologia proposta foi a expansão do modelo TPS para o espaço tridimensional, ou seja, utilizando coordenadas no espaço 3D, e a resolução do sistema de equações (estimativa dos coeficientes do modelo). O modelo TPS 3D, conforme apresentado na seção anterior, se resume à 
solução de um sistema de equações lineares formado pelas Equações 5 e 6. Para efeito desse texto, será utilizada a denominação TPS 2D para se referir à formulação TPS utilizando coordenadas no domínio bidimensional e TPS 3D quando se trata da formulação com coordenadas no domínio tridimensional. Dessa forma, o modelo possibilita a modelagem das distorções utilizando a informação altimétrica, quando disponível, ou independente desta $(\mathrm{h}=0)$. Portanto, o modelo pode ser tratado como sendo 3D, mas modelando um fenômeno de componentes planimétricas.

Como não há redundância na determinação da solução do sistema e a matriz $A$ é quadrada, o vetor dos coeficientes $X$ pode ser encontrado pela multiplicação da matriz inversa dos coeficientes $\left(A^{-1}\right)$ e o vetor das observações $(L)$, na forma $X=A^{-1} L$. A solução dos $(n+4)$ coeficientes (vetor X) para cada componente das coordenadas se resume à solução de um sistema formado por $(n+4)$ equações por $(n+4)$ incógnitas.

Os problemas de condicionamento na solução TPS, decorrentes da geometria das estações do SGB, foram solucionados com a aplicação do limiar de distância de $1 \mathrm{~km}$ para pares de pontos próximos e com a inversão da matriz $A$ pela decomposição em valores singulares (SVD - Singular Value Decomposition). A decomposição SVD é um método muito utilizado para análise e solução de sistema de equações com matrizes singulares ou quase singulares (mal condicionadas). $\mathrm{O}$ método se baseia no seguinte princípio da álgebra linear (PRESS et al., 1992): qualquer matriz $A$ ( $n \times \mathrm{x} u)$, com número de linhas $n$ maior ou igual ao número de colunas $u$, pode ser escrita como um produto de uma matriz coluna ortogonal $U(n \times$ $u$ ), uma matriz diagonal $W(u \times u)$, com elementos nulos ou positivos (valores singulares), e a transposta de uma matriz ortogonal $V(u \times u)$. Portanto:

$$
A=U W V^{T} \text {. }
$$

A matriz diagonal $W$ contém os valores singulares (autovalores) associados à matriz $A$. Os valores contidos em $W$ possibilitam inferências importantes sobre o condicionamento da matriz $A$ e, consequentemente, do sistema de equações. $\mathrm{O}$ cálculo da matriz inversa pela decomposição SVD para $A$ quadrada é dada por:

$$
A^{-1}=V\left[\operatorname{diag}\left(1 / w_{i}\right)\right] U^{T} .
$$

Com os coeficientes do modelo TPS calculados a partir dos pontos homólogos, no caso, entre duas realizações de referenciais geodésicos, a etapa final consiste na aplicação dos coeficientes em pontos na realização de origem, para os quais se deseja conhecer as coordenadas na realização de destino. É importante destacar que todo o processamento foi realizado com coordenadas normalizadas, procurando-se evitar problemas de ordem numérica, dadas as dimensões das coordenadas geodésicas cartesianas ( $\mathrm{X}, \mathrm{Y}$ e $\mathrm{Z}$ ) dos pontos. Após experimentos com diferentes valores adotou-se o intervalo $[-10,10]$, sendo ao final da modelagem realizada a 
normalização inversa para obter as coordenadas geodésicas na mesma escala e magnitude originais.

O fluxograma da Figura 1 ilustra as etapas de determinação do modelo TPS proposto nesse artigo.

Figura 1 - Fluxograma do processo de determinação dos coeficientes do modelo TPS.

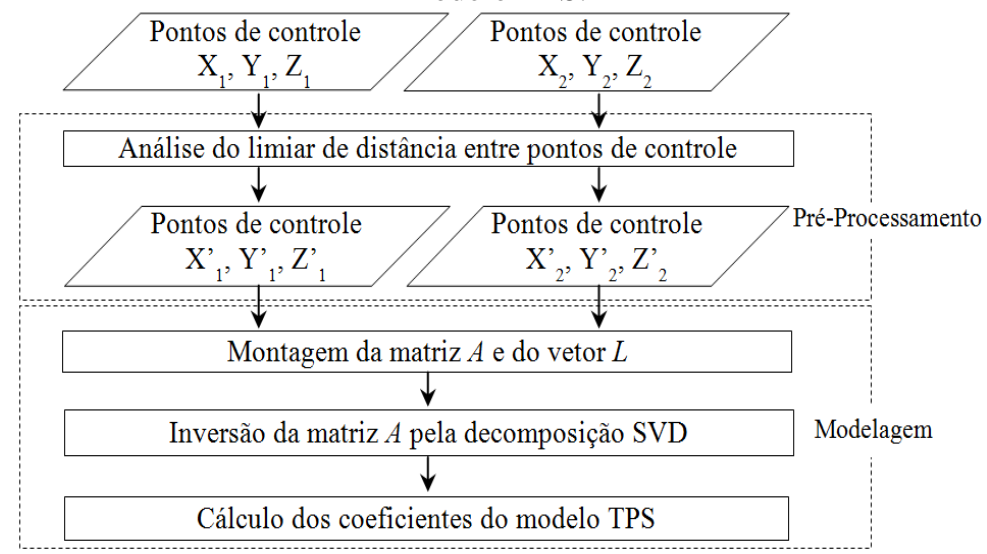

O processo de determinação dos coeficientes do modelo TPS, conforme apresentado na Figura 1, inicia-se com dois conjuntos de pontos de controle homólogos associados às realizações de referenciais geodésicos envolvidos na transformação. Esses pontos passam por uma etapa de pré-processamento no qual são excluídos um dos pontos vizinhos com distâncias iguais ou inferiores ao limiar de distância estabelecido. Os pontos resultantes dessa etapa são utilizados na solução do sistema de equações, ou seja, na determinação dos coeficientes do modelo TPS. A matriz $A$ e o vetor $L$ são montados e aplica-se a decomposição por valores singulares SVD para a inversão da matriz $A$ e posterior determinação do vetor dos parâmetros $\left(X=A^{-1} L\right)$.

Determinado o modelo TPS, seus coeficientes podem ser utilizados para transformar analiticamente as coordenadas da realização de origem para a realização de destino. Este processo de transformação, a exemplo de outros já citados, pode ser realizado basicamente de duas formas: analiticamente, ou seja, utilizando o modelo funcional e aplicando os parâmetros calculados, ou disponibilizando grades de transformação (GT).

\section{EXPERIMENTOS E RESULTADOS}

A validação do método proposto foi realizada a partir de experimentos com dados de estações do SGB, na realização SAD69/96 do sistema SAD69 e SIRGAS2000 do sistema SIRGAS. O modelo TPS foi avaliado considerando: a) o 
conjunto total de pontos homólogos disponíveis nas redes; b) a transformação direta e inversa em pontos não coincidentes com estações do SGB; c) utilizando um conjunto particular de pontos de controle para estimativa dos coeficientes e outro conjunto particular de pontos de verificação para as análises; e d) comparando os resultados do modelo TPS com o ProGriD.

Embora o processo de estimativa dos coeficientes do modelo e de transformação de coordenadas tenha sido realizado com coordenadas geodésicas cartesianas (X, Y e Z), os resultados são apresentados em termos de coordenadas geodésicas curvilíneas (latitude e longitude) por intuitivamente facilitar a análise e compreensão dos resultados. Nestes processamentos foram modeladas distorções planimétricas entre as duas realizações envolvidas, ou seja, considerando a altitude geométrica nula $(\mathrm{h}=0)$.

\subsection{Avaliação do Modelo TPS em Estações do SGB com o Conjunto Total de Pontos Disponível}

Neste experimento, foram determinados os coeficientes do modelo TPS para a transformação entre SAD69/96 e SIRGAS2000 utilizando todo o conjunto de pontos disponível como sendo de controle. O pré-processamento foi realizado nos dados para eliminar pontos próximos, de acordo com o limiar de distância $(1 \mathrm{~km})$. Uma vez estimados os coeficientes do modelo TPS, eles foram aplicados nos mesmos pontos de controle, com o propósito de avaliar a consistência do modelo funcional ao conjunto de dados disponível. As análises se basearam nas discrepâncias entre as coordenadas conhecidas no referencial de destino e as respectivas coordenadas transformadas pelo modelo TPS.

A Figura 2 ilustra as etapas realizadas neste experimento. As etapas relacionadas por linhas pontilhadas representam a aplicação dos coeficientes do modelo TPS em coordenadas fornecidas.

Figura 2 - Fluxograma das etapas do experimento com o conjunto total de pontos.

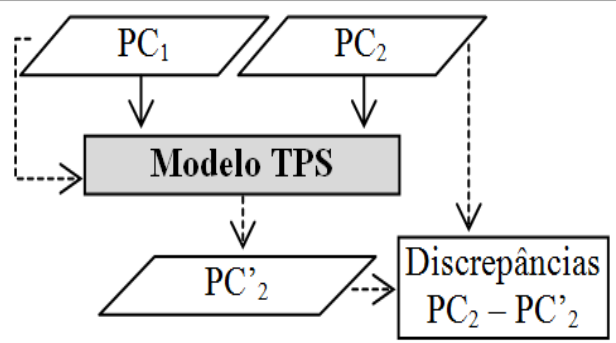

$\mathrm{PC}_{\mathrm{i}}, \mathrm{PC}_{\mathrm{i}}$ : pontos de controle conhecidos e transformados, respectivamente, na realização i. 
O mesmo experimento foi realizado com o ProGriD para fins de comparação entre os modelos. Algumas estatísticas das discrepâncias calculadas nos pontos de controle são apresentadas na Tabela 1.

Tabela 1 - Estatísticas da análise da qualidade da modelagem com a totalidade (4352) dos pontos.

\begin{tabular}{l|c|c|c|c}
\hline \multirow{2}{*}{ Discrepâncias } & \multicolumn{2}{|c|}{ Modelo TPS } & \multicolumn{2}{c}{ ProGriD } \\
\cline { 2 - 5 } & Lat. (m) & Long. (m) & Lat. (m) & Long. (m) \\
\hline Máxima & 0,0074 & 0,0065 & 0,4958 & 0,6457 \\
\hline Mínima & $-0,0103$ & $-0,0082$ & $-1,6711$ & $-0,7656$ \\
\hline Média & 0,0000 & 0,0000 & $-0,0068$ & $-0,0061$ \\
\hline Desvio Padrão & 0,0009 & 0,0006 & 0,0474 & 0,0420 \\
\hline RMSE & 0,0009 & 0,0006 & 0,0479 & 0,0424 \\
\hline $\mathbf{9 0 \%} \leq$ & 0,0012 & 0,0009 & 0,0432 & 0,0448 \\
\hline
\end{tabular}

Conforme a Tabela 1, utilizando o modelo TPS pode-se notar que a discrepância média foi nula tanto em latitude quanto em longitude. Os valores do RMSE foram de $0,9 \mathrm{~mm}$ em latitude e $0,6 \mathrm{~mm}$ em longitude, enquanto que para o ProGriD foram, respectivamente, de 47,9 $\mathrm{mm}$ em latitude e $42,4 \mathrm{~mm}$ em longitude. Em geral, os valores próximos à zero para os indicadores estatísticos sugerem que a modelagem atende à característica desejada para o modelo, que deve garantir a fidelidade dos valores nos pontos amostrais.

Comparando ainda os resultados TPS versus ProGriD, verifica-se a redução superior a $97 \%$ em todos os indicadores estatísticos na modelagem por TPS, evidenciando que a modelagem proposta se ajusta melhor aos pontos de controle que o ProGriD. Em 90\% dos pontos transformados pelo modelo TPS as discrepâncias máximas foram inferiores a $1,2 \mathrm{~mm}$ em latitude e $0,9 \mathrm{~mm}$ em longitude, já com o ProGriD os valores foram de 43,2 $\mathrm{mm}$ e 44,8 $\mathrm{mm}$ em cada componente, respectivamente.

\subsection{Avaliação das Transformações Direta e Inversa em Pontos não Coincidentes com Estações do SGB}

A análise da qualidade das transformações direta e inversa por meio da metodologia proposta é realizada neste experimento. Para tanto, foram utilizados 368 pontos regularmente espaçados de $2^{\circ} \times 2^{\circ}$ em latitude e longitude para verificação, cujas coordenadas foram definidas matematicamente (simuladas), estando ainda associadas ao Sistema SAD69 (notar que nesse caso o sistema e rede não apresentam diferenças, por serem coordenadas matemáticas). Esses pontos foram convertidos para o SIRGAS2000 aplicando o modelo TPS obtido com o conjunto total de pontos. As coordenadas resultantes da transformação foram novamente convertidas para o sistema SAD69/96 usando o modelo TPS inverso, ou seja, utilizando a realização SIRGAS2000 como origem. Uma vez que as 
coordenadas iniciais dos pontos utilizados estavam isentas de erros, em função da maneira como foram definidas (matematicamente), as diferenças entre as coordenadas originais e as transformadas representam os erros decorrentes das próprias transformações. Este mesmo experimento também foi realizado usando o ProGriD. A Figura 3 ilustra as etapas deste experimento em um fluxograma e a Figura 4 mostra a distribuição dos pontos utilizados.

Figura 3 - Fluxograma do experimento onde é feita a comparação das transformações direta e inversa.

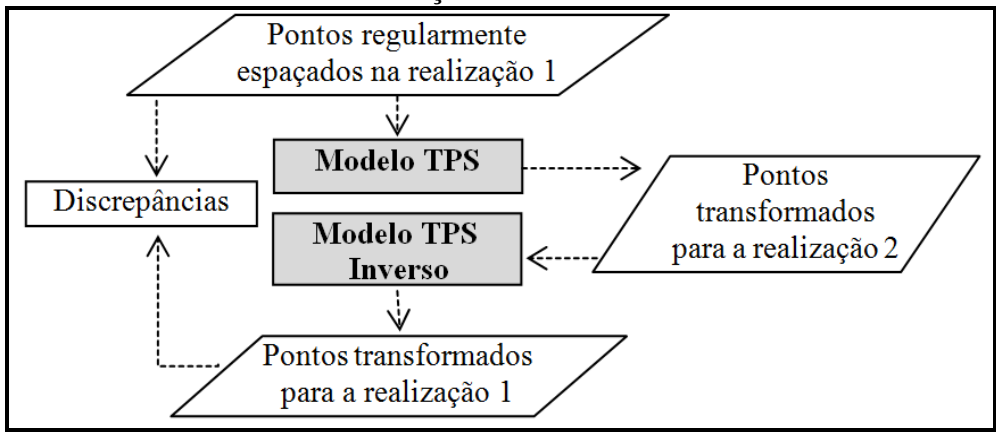

Figura 4 - Distribuição dos pontos para o experimento de transformação direta e inversa.



Bol. Ciênc. Geod., sec. Artigos, Curitiba, v. 20, nº 1, p.19-38, jan-mar, 2014. 
A Tabela 2 apresenta os resultados das discrepâncias entre as coordenadas originais e transformadas nos pontos de verificação utilizando tanto o modelo TPS quanto o sistema ProGriD.

Tabela 2 - Estatísticas das discrepâncias na transformação direta e inversa após a modelagem por TPS.

\begin{tabular}{l|c|c|c|c}
\hline \multirow{2}{*}{ Discrepâncias } & \multicolumn{2}{|c|}{ Modelo TPS } & \multicolumn{2}{c}{ ProGriD } \\
\cline { 2 - 5 } & Lat. (m) & Long. (m) & Lat. (m) & Long. (m) \\
\hline Máxima & 0,0035 & 0,0018 & 0,0000 & 0,0000 \\
\hline Mínima & $-0,0034$ & $-0,0026$ & $-0,0011$ & $-0,0011$ \\
\hline Média & 0,0000 & 0,0000 & $-0,0002$ & $-0,0002$ \\
\hline Desvio Padrão & 0,0005 & 0,0003 & 0,0005 & 0,0004 \\
\hline $\mathbf{9 0 \%} \leq$ & 0,0005 & 0,0003 & 0,0011 & 0,0010 \\
\hline
\end{tabular}

O valor máximo absoluto de erro esperado para a modelagem com TPS é de aproximadamente $3,5 \mathrm{~mm}$ em latitude e $2,6 \mathrm{~mm}$ em longitude. Os demais valores são da ordem dos décimos de milímetro. Comparando os resultados obtidos pelo modelo TPS e ProGriD é possível verificar que ambos os modelos proporcionaram discrepâncias inferiores a 3,5 mm. Em termos de discrepâncias máximas e mínimas, o ProGriD resultou em valores menores que o modelo TPS, inferiores a 1,1 mm em cada componente. Quanto às discrepâncias médias, desvios padrão em latitude e discrepâncias em $90 \%$ dos pontos, os dois modelos mostraram resultados similares. No caso do desvio padrão em longitude, discrepância em $90 \%$ dos pontos e discrepâncias médias nas duas componentes o modelo TPS resultou em valores menores que o ProGriD.

As Figuras 5 e 6 apresentam uma comparação entre os resultados obtidos pelo modelo TPS e pelo ProGriD com relação às estatísticas apresentadas na Tabela 2.

Figura 5 - Comparação entre TPS e ProGriD em latitude.

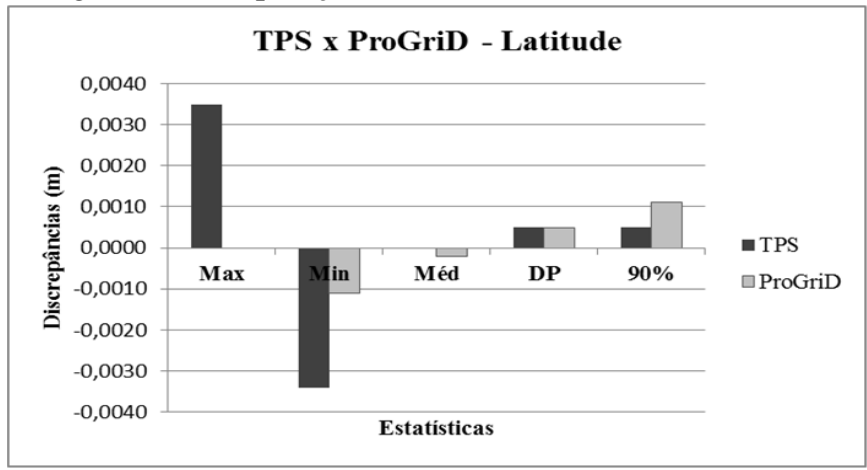

Bol. Ciênc. Geod., sec. Artigos, Curitiba, v. 20, nº 1, p.19-38, jan-mar, 2014. 
Figura 6 - Comparação entre TPS e ProGriD em longitude.

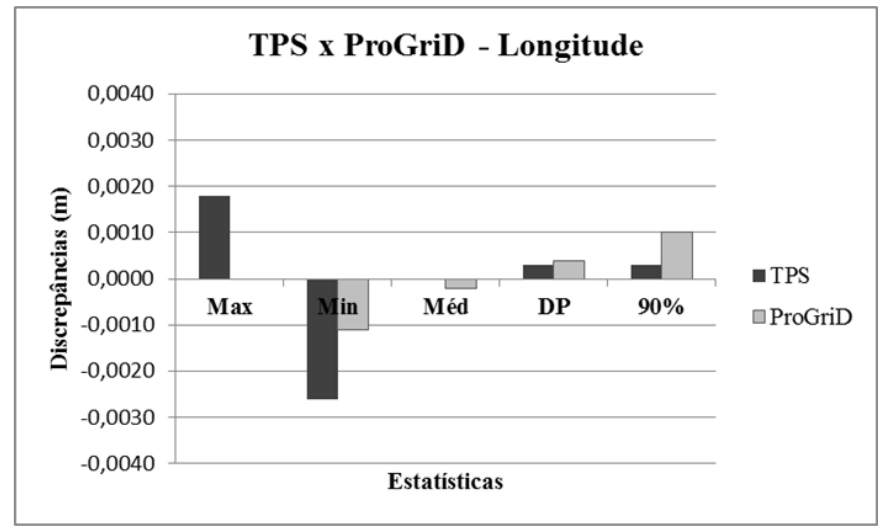

É possível verificar pelas Figuras 5 e 6 que o ProGriD proporciona menores valores absolutos de discrepâncias máximas e mínimas que o TPS neste experimento. As maiores discrepâncias nos pontos de verificação obtidas pelo modelo TPS ocorreram exclusivamente em regiões de extrapolação ou com carência de pontos, como é o caso das divisas entre MT, GO e TO e na região sudoeste do MS. Este experimento indica que, assumindo como desprezível a discrepância máxima de 3,5 mm, para uma rede geodésica desta dimensão, ambos os modelos são coerentes entre si.

\subsection{Avaliação da Qualidade da Modelagem em Pontos de Verificação}

Esse experimento tem por objetivo avaliar a qualidade da modelagem por TPS nos pontos de verificação de estações do SGB. Para isso foram utilizados os pontos de cálculo $\left(\mathrm{PC}_{1}, \mathrm{PC}_{2}\right)$ em duas realizações para estimar os coeficientes do modelo TPS e pontos de verificação $\left(\mathrm{PV}_{1}, \mathrm{PV}_{2}\right)$, distintos dos pontos de cálculo, para avaliar a qualidade da transformação. Estimados os coeficientes do modelo TPS nos pontos de cálculo, aplicou-se a modelagem nos pontos de verificação e foram comparadas as coordenadas de referência (ajustadas pelo IBGE em SIRGAS2000) com as coordenadas transformadas com o modelo TPS. A Figura 7 apresenta um fluxograma com as etapas realizadas neste experimento.

Pode-se dizer que as discrepâncias obtidas para as coordenadas representam a acurácia das mesmas, já que as coordenadas transformadas para a realização de destino foram comparadas com os valores de referência, ou seja, as próprias coordenadas no sistema de destino. 
Figura 7 - Fluxograma das etapas do experimento com pontos de verificação independentes.

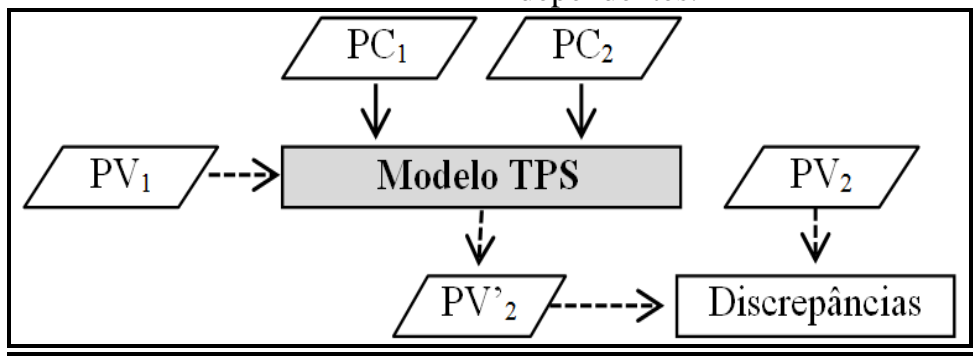

O conjunto total de dados disponível na realização SAD69/96 é composto por 4.474 pontos (técnicas clássicas), sendo 4.067 pontos de controle e 407 pontos de verificação. Foram descartados 99 pontos do conjunto de pontos de controle pelo limiar de distância. É importante salientar que o número de pontos descartados neste experimento e no primeiro são diferentes, uma vez que a avaliação do limiar é feita nos pontos utilizados como controle. No primeiro experimento todos os pontos disponíveis foram utilizados como controle, enquanto que neste experimento foram utilizados cerca de $90 \%$ do conjunto total de pontos como pontos de controle. A distribuição dos pontos (técnicas clássicas) da realização SAD69/96 utilizados neste experimento pode ser visualizada na Figura 8.

Figura 8 - Distribuição dos pontos de controle e de verificação da realização SAD69/96.



Na Tabela 3 são apresentadas as informações estatísticas das discrepâncias nos pontos de verificação antes e após a modelagem por TPS. 
Tabela 3 - Discrepâncias nos pontos de verificação antes e após a modelagem TPS.

\begin{tabular}{l|c|c|c|c}
\hline \multirow{2}{*}{ Discrepâncias } & \multicolumn{2}{|c|}{ Antes da Modelagem } & \multicolumn{2}{c}{ Após a Modelagem por TPS } \\
\cline { 2 - 5 } & Lat. (m) & Long. $(\mathbf{m})$ & Lat. $(\mathbf{m})$ & Long. $(\mathbf{m})$ \\
\hline Máxima & $-37,5658$ & $-35,4456$ & 0,5482 & 0,4795 \\
\hline Mínima & $-56,6398$ & $-56,2023$ & $-0,5109$ & $-0,2965$ \\
\hline Média & $-49,9696$ & $-45,7392$ & 0,0080 & 0,0012 \\
\hline Desvio Padrão & 3,9509 & 4,5453 & 0,0779 & 0,0676 \\
\hline RMSE & 53,7011 & 46,8038 & 0,0782 & 0,0675 \\
\hline $\mathbf{9 0 \%} \leq$ & 54,5998 & 50,5703 & 0,0256 & 0,0067 \\
\hline $\mathbf{N}^{\circ}$ de pontos & \multicolumn{4}{|c}{407} \\
\hline
\end{tabular}

As discrepâncias calculadas nos pontos de verificação antes da modelagem foram obtidas subtraindo das coordenadas conhecidas (ajustadas) em SIRGAS2000 as coordenadas conhecidas em SAD69/96. A magnitude das discrepâncias obtidas antes da modelagem é da ordem de $50 \mathrm{~m}$ em latitude e $46 \mathrm{~m}$ em longitude, com respectivos desvios padrão de $3,9 \mathrm{~m}$ e $4,5 \mathrm{~m}$. Após a modelagem por TPS, as discrepâncias médias foram reduzidas para $8 \mathrm{~mm}$ e $1,2 \mathrm{~mm}$, com desvios padrão de $78 \mathrm{~mm}$ e $68 \mathrm{~mm}$, respectivamente, em latitude e longitude. Os valores de RMSE foram de 78,2 $\mathrm{mm}$ em latitude e $67,5 \mathrm{~mm}$ em longitude. Em termos de distorções, as Figuras 9 e 10 representam os vetores de distorção nos pontos de verificação antes e após a modelagem por TPS, respectivamente.

Figura 9 - Distorções nos pontos de verificação, antes da modelagem.

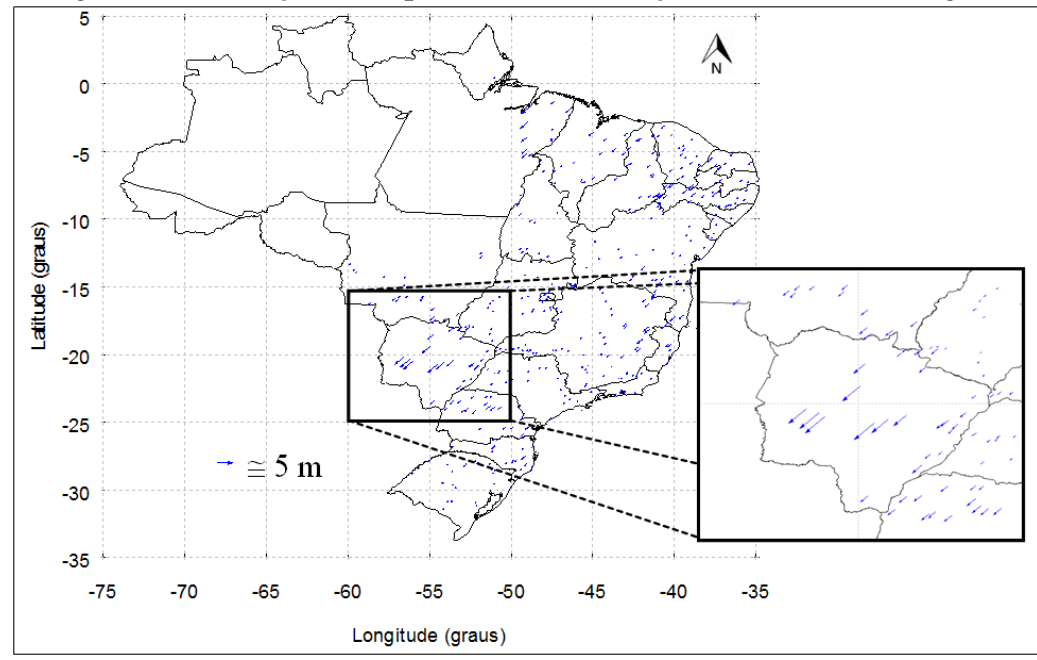

Bol. Ciênc. Geod., sec. Artigos, Curitiba, v. 20, nº 1, p.19-38, jan-mar, 2014. 
Figura 10 - Distorções nos pontos de verificação, após a modelagem por TPS.

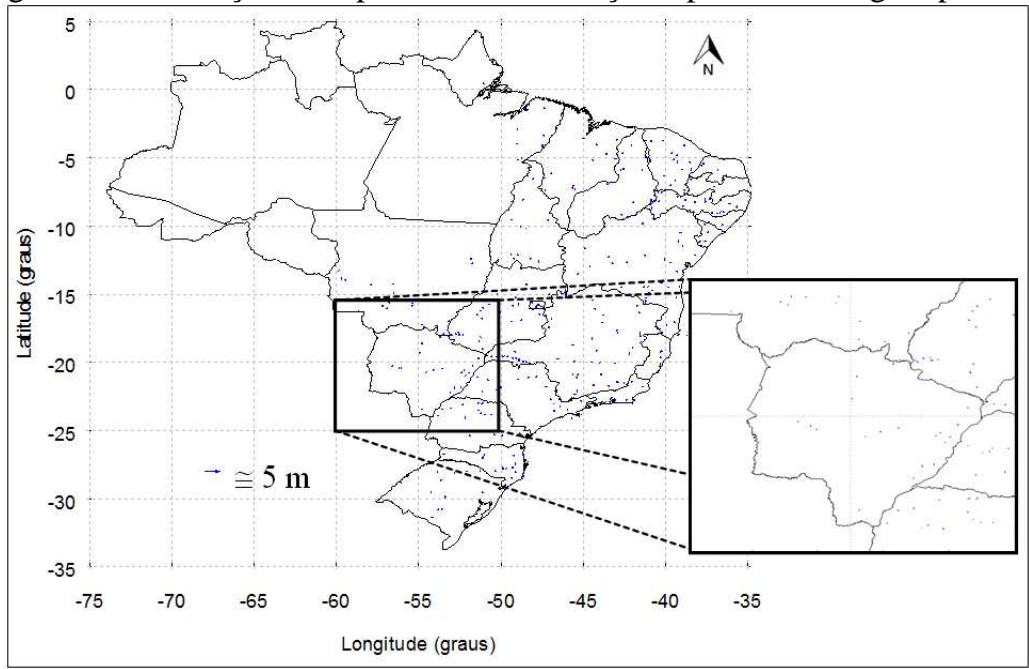

Nota-se na Tabela 3 que a magnitude do RMSE é influenciada pela discrepância de poucos pontos de verificação localizados nas regiões onde a modelagem é mais complexa e as discrepâncias são relativamente superiores aos demais pontos. A discrepância elevada em poucos pontos da realização SAD69/96 é comprovada se analisados os valores de discrepância em $90 \%$ dos pontos, os quais são inferiores a $25,6 \mathrm{~mm}$ em latitude e $6,7 \mathrm{~mm}$ em longitude. Mais detalhes são apresentados em Magna Júnior (2012).

\section{CONSIDERAÇÕES FINAIS E CONCLUSÕES}

A transformação de coordenadas entre realizações de referenciais geodésicos é uma necessidade mundial e recorrente, sobretudo com a rápida expansão e popularização de métodos de posicionamento de melhor acurácia. Procurou-se, neste artigo, colaborar com o processo de transformação de referenciais propondo uma metodologia baseada em Thin-Plate Splines. O método proposto realiza a transformação no espaço tridimensional, tratando conjuntamente a modelagem de distorções inerentes às diferentes realizações.

Para a determinação dos coeficientes do modelo foi aplicada a formulação TPS $3 \mathrm{D}$, a qual preserva as mesmas características de continuidade e suavidade do modelo TPS convencional. A validação da metodologia se deu por meio de experimentos com dados tanto simulados quanto reais, sendo este último obtido a partir de dados das estações do SGB em SAD69/96 e SIRGAS2000, sendo também realizada a comparação com o ProGriD.

Em função de problemas de condicionamento na modelagem por TPS decorrentes da geometria da rede, foi estabelecido um limiar mínimo de distância 
fixado em $1 \mathrm{~km}$ para os pontos utilizados na estimativa do modelo e utilizada a técnica SVD para a inversão da matriz dos coeficientes.

$\mathrm{Na}$ análise das discrepâncias com o conjunto total de pontos, os valores de RMSE obtidos foram de $0,9 \mathrm{~mm}$ em latitude e de $0,6 \mathrm{~mm}$ em longitude. Os resultados indicam que o modelo TPS se ajusta adequadamente aos pontos de controle, ou seja, proporciona discrepâncias próximas a zero nestes pontos.

A qualidade da modelagem foi verificada nas transformações direta e inversa entre as realizações SAD69/96 e SIRGAS2000, com pontos não coincidentes com estações do SGB. Os resultados mostraram que o erro máximo esperado para a modelagem com TPS é de aproximadamente 3,5 mm em latitude e 2,6 mm em longitude. Em $90 \%$ dos pontos os valores de discrepância foram inferiores a 0,5 mm em latitude e $0,3 \mathrm{~mm}$ em longitude. Os valores obtidos representam o erro inerente ao próprio processo de transformação, já que as coordenadas utilizadas neste experimento foram obtidas matematicamente e isentas de erros.

$\mathrm{Na}$ avaliação da qualidade da modelagem nos pontos de verificação independentes, o RMSE obtido após a modelagem foi de 78,2 $\mathrm{mm}$ em latitude e $67,5 \mathrm{~mm}$ em longitude, evidenciando uma redução superior a $99 \%$ nas distorções em cada componente. Em $90 \%$ dos pontos as discrepâncias foram inferiores a 25,6 $\mathrm{mm}$ em latitude e $6,7 \mathrm{~mm}$ em longitude. Em razão das discrepâncias serem calculadas utilizando como referência os valores ajustados (reais), pode-se dizer que os resultados (RMSE) expressam a acurácia da transformação nos pontos de verificação.

Na comparação do modelo TPS com o ProGriD, verificou-se que a modelagem por TPS proporciona menores discrepâncias nos pontos de controle após a transformação das coordenadas. Em $90 \%$ dos pontos de controle as discrepâncias calculadas foram inferiores a 1,2 $\mathrm{mm}$ e $0,9 \mathrm{~mm}$ para o modelo TPS e 43,2 $\mathrm{mm}$ e $44,8 \mathrm{~mm}$ para o ProGriD, respectivamente, em latitude e longitude. Os valores de RMSE foram de 0,9 mm e 0,6 mm com o modelo TPS e 43,2 $\mathrm{mm}$ e 44,8 $\mathrm{mm}$ com o ProGriD. Foi possível observar que ao utilizar o modelo TPS todos os indicadores estatísticos considerados sofreram redução superior a 97\% em seus valores.

Em função dos resultados apresentados, conclui-se que a modelagem por TPS mostrou-se eficiente na transformação de coordenadas e na modelagem das distorções entre realizações de referenciais geodésicos, proporcionando uma transformação de qualidade e que garante integridade aos dados transformados. A partir das análises realizadas e dos resultados obtidos, considera-se que a modelagem por TPS é um método promissor. Adicionalmente, destaca-se que do modo como o método foi apresentado, ele está preparado para incorporar a informação altimétrica, quando disponível, sem a necessidade de adaptações, já que ele utiliza como dados de entrada as coordenadas geodésicas cartesianas.

\section{AGRADECIMENTOS}

Os autores agradecem à Fundação Instituto Brasileiro de Geografia e Estatística (IBGE), ao GT3, pelos dados fornecidos, e à Coordenação de Aperfeiçoamento de 
Pessoal de Nível Superior (CAPES) pela bolsa de doutorado concedida durante parte do desenvolvimento deste trabalho.

\section{REFERÊNCIAS}

BOOKSTEIN, F. L. Principal warps: Thin-plate splines and the decomposition of deformations. IEEE Transactions on Pattern Analysis and Machine Intelligence, v. 11, n. 6, p. 567-585, jun. 1989.

CASTANHO, J. E. C. Registro de Imagens: uma solução para a modelagem de deformações e automação da correspondência. 1997. 153 p. Tese (Doutorado em Engenharia Elétrica) - Faculdade de Engenharia Elétrica e de Computação, Universidade Estadual de Campinas, Campinas.

COSTA, S. M. A Integração da rede geodésica brasileira aos sistemas de referência terrestres. 1999. 170 p. Tese (Doutorado em Ciências Geodésicas) Programa de Pós-Graduação em Ciências Geodésicas, Universidade Federal do Paraná, Curitiba.

IBGE - FUNDAÇÃO INSTITUTO BRASILEIRO DE GEOGRAFIA E ESTATÍSTICA. ProGriD - guia do usuário. Rio de Janeiro, 2009. Disponível em: <ftp://geoftp.ibge.gov.br/documentos/geodesia/ projeto_mudanca_ referencial_geodesico/progrid_guia_do_usuario.pdf >. Acesso em: 10 set. 2012.

. Resolução $\mathrm{n}^{\circ} 1$, de 2005. Altera a caracterização do sistema geodésico brasileiro, Rio de Janeiro, fev. 2005. Disponível em: <ftp://geoftp.ibge. gov.br/documentos/geodesia/projeto_

mudanca_referencial_geodesico/legislacao/rpr_01_25fev2005.pdf >. Acesso em: 10 set. 2012.

- Resolução $\mathrm{n}^{\circ}$ 22, de 1983. Especificações e normas gerais para levantamentos geodésicos, Rio de Janeiro, 1983.

JUNKINS, D.; ERICKSON, C. Version 2 of the National Transformation Between NAD27 and NAD83 and its Importance for GPS Positioning in Canada. Draft Report, Geodetic Survey Division, Geomatics Canada, 1996.

MAGNA JÚNIOR, J. P. Modelagem de distorções entre realizações de referenciais geodésicos. 2007. 108 f. Dissertação (Mestrado em Ciências Cartográficas) Faculdade de Ciências e Tecnologia, Universidade Estadual Paulista, Presidente Prudente.

. Modelagem de distorções entre SAD 69 e SIRGAS 2000 pelo método de Shepard e grades regulares. Boletim de Ciências Geodésicas, Curitiba, v. 15, n. 3, p. 388-409, jul.-set. 2009.

. $O$ uso de thin-plate splines na transformação de coordenadas com modelagem de distorções entre realizações de referenciais geodésicos. 2012. 117 f. Tese (Doutorado em Ciências Cartográficas) - Faculdade de Ciências e Tecnologia, Universidade Estadual Paulista, Presidente Prudente. 
OLIVEIRA, L. C. Realizações do Sistema Geodésico Brasileiro associadas ao SAD 69 - uma proposta metodológica de transformação. 1998. 197 p. Tese (Doutorado em Engenharia) - Escola Politécnica, Universidade de São Paulo, São Paulo.

OLIVEIRA, L. C.; SANTOS, M. C.; NIEVINSKI, F. G.; LEANDRO, R. F.; COSTA, S. M. A.; SANTOS, M. F.; MAGNA JÚNIOR, J. P.; GALO, M.; CAMARGO, P. O.; MONICO, J. F. G.; SILVA, C. U.; MAIA, T. B. Searching for the Optimal Relationships Between SIRGAS2000, South American Datum of 1969 and Córrego Alegre in Brazil. In: SIDERIS, M. G. Observing our Changing Earth. Itália: Springer Berlin Heidelberg, 2008. v. 133, 71-79.

PINO, L. M.; FIRKOWSKI, H. Avaliação de modelos de transformação bidimensional para a compatibilização de bases cartográficas associadas a diversos sistemas geodésicos de referência. Boletim de Ciências Geodésicas, Curitiba, v. 15, n. 2, p. 259-276, abr.-jun. 2009.

PRESS, W. H.; TEUKOLSKY, S. A.; VETTERLING, W. T.; FLANNERY, B. P. Numerical recipes in $C$ : the art of scientific computing. 2nd ed. Cambrigde: Cambridge University Press, 1992. p. 994.

TSAI, Y.; LIN, H.; HU, Y.; YU, C.; LIN, K. Thin-plate spline technique for medical image deformation. Journal of medical and biological engineering, $\mathrm{v}$. 20, n. 4, p. 203-210, out. 2000.

(Recebido em abril de 2012. Aceito em março de 2013). 